\title{
MiR-206 inhibits reorganization of the cytoskeleton in melanoma cells by targeting DDX5
}

\author{
Shenglin $\mathrm{Wu}^{1}$, Shan $\mathrm{Nie}^{2}$, Jian Wang ${ }^{3 *}$ \\ ${ }^{1}$ Department of Burn, Plastic Surgery, and Wound Repair, The People's Hospital of LiShui, Lishui, Zhejiang Province 323000 , \\ ${ }^{2}$ Department of Stomatology, First Hospital of Nanchang City, ${ }^{3}$ Department of Dermatology, The First Affiliated Hospital of \\ Nanchang University, Nanchang City, Jiangxi Province 330006, China
}

*For correspondence: Email: jianw2570@163.com; Tel: +86-0791-8692745

Sent for review: 9 August 2021

Revised accepted: 27 October 2021

\begin{abstract}
Purpose: To investigate the role and mechanism of microRNA-206 (miR-206) in cytoskeleton reorganization in melanoma cells.

Methods: MiR-206 and RNA helicase p68 (DDX5) expression levels were measured in A375, A875, and HEM-M cells by quantitative real time polymerase chain reaction ( $q R T-P C R$ ). A DDX5 overexpression cell line was constructed, and DDX5 overexpression, A375, and A875 cells were transfected with miR-206 mimic or DDX5 small interfering RNA (siRNA). Transwell assay was used to assess cell migration and invasion of $A 375$ and $A 875$ cells, while Luciferase reporter assay was used to determine the putative target of miR-206. DDX5, miR-206, vinculin, coronin3, and ezrin expression levels were evaluated by $q R T-P C R$. Protein expressions of DDX5, vinculin, coronin3, and ezrin were evaluated by western blot analysis.

Results: DDX5 expression was higher and miR-206 expression lower in A375 and A875 cells when compared to HEM-M cells ( $p<0.05$ ). Knockdown of DDX5 and overexpression of miR-206 repressed invasion and migration, and inhibited expression of vinculin, coronin3, and ezrin in A375 and A875 cells $(p<0.05)$. However, overexpression of DDX5 reversed the effect of miR-206 on cytoskeletal protein expression. Luciferase reporter assay data confirmed that DDX5 is a direct target of miR-206 $(p<0.05)$. Conclusion: MiR-206 suppresses reorganization of the cytoskeleton in melanoma cells by targeting $D D X 5$, and is thus, a promising target for the treatment of melanoma.
\end{abstract}

Keywords: Melanoma, MicroRNA-206, Cytoskeleton reorganization, RNA helicase 068

\begin{abstract}
This is an Open Access article that uses a funding model which does not charge readers or their institutions for access and distributed under the terms of the Creative Commons Attribution License (http://creativecommons.org/licenses/by/4.0) and the Budapest Open Access Initiative (http://www.budapestopenaccessinitiative.org/read), which permit unrestricted use, distribution, and reproduction in any medium, provided the original work is properly credited.

Tropical Journal of Pharmaceutical Research is indexed by Science Citation Index (SciSearch), Scopus, International Pharmaceutical Abstract, Chemical Abstracts, Embase, Index Copernicus, EBSCO, African Index Medicus, JournalSeek, Journal Citation Reports/Science Edition, Directory of Open Access Journals (DOAJ), African Journal Online, Bioline International, Open-J-Gate and Pharmacy Abstracts
\end{abstract}

\section{INTRODUCTION}

Malignant melanoma results from proliferation of abnormal melanocytes, has become a common malignant tumor in the past decades, and its incidence and mortality rate are rising [1]. Exposure to the sun and genetic factors are risk factors for melanoma progression [1]. Surgery and traditional first-line treatments do not prolong survival, and there is no preventative vaccine [2]. In addition, the underlying mechanism of melanoma development and metastasis has not been clarified yet. Therefore, it is necessary to elucidate the biological mechanism of melanoma to develop a promising therapeutic remedy. 
MicroRNAs (miRNAs), common exosomal constituents, have been implicated in diverse biological processes through their effects on various target genes [3]. It was shown that miRNA affects the cytoskeletal reorganization of cancer cells and regulates the actin cytoskeleton [4]. miR-206 was found to suppress cell migration and affect the actin cytoskeleton and cell morphology by targeting Coronin $1 \mathrm{C}$ in triplenegative breast cancer [5] . Moreover, miR-206 inhibited cell invasion and migration of MDA-MB231 breast cancer cells partially through regulation of actin cytoskeleton reorganization, especially filopodia formation []ㅡ. However, the effect of miR-206 on actin cytoskeleton reorganization, cell morphology, and migration in melanoma cells has not been reported.

The RNA helicase DDX5, also known as RNA helicase p68, belongs to a family of highly conserved proteins involved in tumorigenesis, metastasis, and proliferation of many human malignancies [7]. Abnormal expression of DDX5 has been observed in various cancers [으, $\underline{9}$. DDX5 contributed to cytoskeletal reorganization in basal breast cancer cells via modulation of the DDX5-miR-182-actin cytoskeleton pathway [10]. However, the role of DDX5 in cytoskeletal reorganization in melanoma cells remains unclear. In this study, the effects of overexpression and knockdown of miR-206 and DDX5 and their interactions were analyzed in A375 and A875 melanoma cells.

\section{EXPERIMENTAL}

\section{Cell culture}

A375, A875, and HEM-M cell lines were obtained from the Cell Bank of the Chinese Academy of Sciences (Shanghai, China) and maintained in RPMI-1640 medium (Gibco, USA) containing $10 \%$ fetal bovine serum (Gibco, USA) with $5 \%$ $\mathrm{CO}_{2}$ at $37^{\circ} \mathrm{C}$.

\section{DDX5 expression construct}

cDNA encoding DDX5 was amplified by polymerase chain reaction (PCR) and subcloned into an overexpression vector (Invitrogen, USA). The primers used to amplify and clone DDX5 are shown in Table 1. Subsequently, Lipofectamine 2000 (Thermo Fisher, USA) was used to transfect the DDX5 overexpression plasmid into A375 and A875 cells according to the manufacturer's instructions.

\section{Cell transfection}

Homo sapiens (hsa)-miR-206, an miR-206 mimic (Qiagen, Germany), or a negative control (NC) mimic (Qiagen, Germany) was transfected into A375 cells, A875 cells, and the DDX5 overexpression cell line. To evaluate knockdown of DDX5, a duplex DDX5 siRNA oligonucleotide (Invitrogen, USA) or an RNA interference negative control (Invitrogen, USA) was transfected into A375 and A875 cells with Lipofectamine 2000 (Invitrogen, USA) and TransIT-LT1 Transfection Reagent for $48 \mathrm{~h}$.

\section{Luciferase reporter assay}

Target genes were predicted using Targetscan (http://www.targetscan.org), and the 3'-UTR of DDX5 was cloned into the psicheck2 plasmid to create psicheck2-DDX5-WT. Then, the miRNA seed sequence for DDX5 was mutated to create psicheck2-DDX5-MUT. Subsequently, psicheck2-DDX-WT or psicheck2-DDX-MUT were co-transfected with a miR-206 mimic or the NC mimic using Lipofectamine 2000 (Invitrogen). A dual-reporter luciferase assay system (Switchgear Genomics) was used to measure expression $24 \mathrm{~h}$ after transfection. Firefly luciferase activity was normalized to Renilla luciferase activity.

\section{Transwell assay}

After transfection of $\mathrm{A} 375$ and $\mathrm{A} 875$ cells with DDX5 siRNA, miR-206 mimic, or the NC mimic for $24 \mathrm{~h}$, the cells were harvested and washed once with PBS (Invitrogen, USA). Next, $8 \mu \mathrm{m}$ pore inserts (Costar, High Wycombe, UK), plain inserts (for migration), or matrigel-coated inserts (for invasion) were placed into the wells of 24well culture plates to separate the upper and lower chambers. The upper chamber was seeded with $1 \times 10^{5}$ cells and the lower chamber was filled with serum-free medium. Extracellular matrix gel was used in the cell invasion assay.

Table 1: DDX amplification and cloning primers

\begin{tabular}{|c|c|c|}
\hline \multirow[t]{2}{*}{ Gene } & \multicolumn{2}{|c|}{ Amplification and cloning primers } \\
\hline & Forward & Reverse \\
\hline DDX5 & $\begin{array}{l}\text { 5'- } \\
\text { CGGATCCACCGCAACCATTG } \\
\text { ACGCC-3' }\end{array}$ & $\begin{array}{l}\text { 5'-- } \\
\text { GGGATCCTTACTTATCATCGTCGTCCTTG } \\
\text { TAGTCTTGGGAATATCCTGTTGGC-3' }\end{array}$ \\
\hline
\end{tabular}


Finally, after $48 \mathrm{~h}$ of incubation, cells that migrated and invaded were stained with hematoxylin and eosin and quantified by light microscopy (Olympus Corporation, Tokyo, Japan).

\section{Quantitative real-time reverse transcriptase- polymerase chain reaction (qRT-PCR)}

Total RNA was exacted using TRIzol reagent (Life Technologies, Carlsbad, CA, USA) and reverse-transcribed into cDNA using the RNeasy kit (Qiagen, Valencia, CA) and the Omniscript reverse transcription kit (Qiagen). Real-time quantitative PCR was performed with the SYBR Green PCR kit (Takara, Dalian, China) and the LightCycler 480 System (Roche Applied Science) with a thermocycle profile of $95^{\circ} \mathrm{C}$ for $10 \mathrm{~min}$ and then 40 cycles of $95^{\circ} \mathrm{C}$ for $10 \mathrm{~s}$ and $60^{\circ} \mathrm{C}$ for 20 s. Gene expression was normalized to U6 snRNA expression using the $2^{-\triangle \Delta C T}$ method [11]. Primers used for qRT-PCR are shown in Table 2.

\section{Western blot analysis}

Cells were pelleted and lysed using lysis buffer containing protease and phosphatase inhibitors (Thermo Fisher Scientific, USA). Protein concentrations of cell extracts were quantified with a BCA Protein Assay kit (Thermo Fisher Scientific, USA). Proteins were separated by 10 $\%$ SDS-PAGE, transferred to polyvinylidene difluoride membranes, and the membranes were incubated with primary antibodies against DDX5 (ab126730, Abcam, 1:400), vinculin (ab129002, Abcam, 1:10000), coronin3 (ab15719, Abcam, 1:10000), and ezrin (ab40839, Abcam, 1:2000) overnight at $4^{\circ} \mathrm{C}$. Then, the membranes were incubated with anti-rabbit IgG antibody (GE Healthcare, 1:10000) and visualized using an enhanced chemiluminescent detection system (Millipore, CA).

\section{Statistical analysis}

SPSS software (version 18.0, Chicago, IL) was used for statistical analysis. Data were presented as mean \pm standard deviation. One-way ANOVA with the Tukey's post-hoc test was utilized to compare multiple groups. $p<0.05$ was considered statistically significant.

\section{RESULTS}

\section{DDX5 expression increased and miR-206 expression decreased in malignant melanoma cells}

qPCR analysis showed that DDX5 expression was higher in A375 and A875 cells than in HEMM cells ( $p<0.01$; Figure 1A), whereas miR-206 was lower in A375 and A875 cells than in HEM$M$ cells $(p<0.01$; Figure 1B). These data indicate that DDX5 and miR-206 expression differs in malignant melanoma cells and normal human epidermal melanocytes.
A

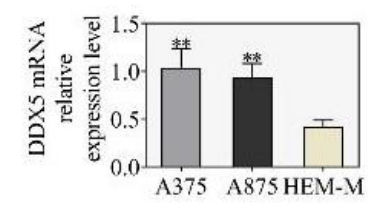

B

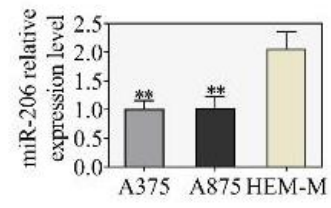

Figure 1. qPCR analysis showed that DDX5 and miR206 were differentially expressed in malignant melanoma cells and normal human epidermal melanocytes. A, DDX5 expression was higher in A375 and A875 cells than in HEM-M cells. B, miR-206 expression was lower in A375 and A875 cells than in HEM-M cells. ${ }^{* *} p<0.01$, compared to HEM-M cells

\section{MiR-206 inhibits cell invasion and migration of malignant melanoma cells}

To investigate the role of DDX5 in melanoma cells, DDX5 siRNA was used to knock down DDX5 expression in A375 and A875 cells, and this knockdown was confirmed by qPCR analysis and western blot $(p<0.01$; Figure 2A, B). In addition, a miR-206 mimic was used to overexpress miR-206 in A375 and A875 cells, and this overexpression was confirmed by qPCR analysis ( $p<0.01$; Figure 2C). Transwell assays showed that knockdown of DDX5 and overexpression of miR-206 repressed invasion and migration of $\mathrm{A} 375$ and $\mathrm{A} 875$ cells when compared to the NC siRNA or NC miR-206 groups $(p<0.01$; Figure 2D). Thus, miR-206

Table 2: qRT-PCR primer sequences

\begin{tabular}{lll}
\hline Gene & Forward primer & \multicolumn{2}{c}{ Reverse primer } \\
\hline DDX5 & 5'-ACAGAATTTCACTGAACCCACTGC-3 & 5'-GACAATGGCAGGAAGCAAATAAGA-3' \\
miR-206 & 5'-AGCTCGATTAAGGTGGAATGTAAGGAAGT-3' & 5'-CTCAACTGGTGTCGTGGAGTCGG-3' \\
Vinculin & 5'- TGAGCTTGCTCCTCCCAAAC-3' & 5'-CTGCCTCAGCTACAACACCT- \\
Coronin3 & 5'-CTGCACAGCTTCCAAAGACAAGA-3 & 5'-GGCTGAACCCAGTGGTGAAGA-3' \\
Ezrin & 5'-ACCATGGATGCAGAGCTGGAG-3' & 5'-ACATAGTGGAGGCCAAAGTACCACA-3 \\
GAPDH & 5'-GGTGAAGGTCGGAGTCAACGG-3' & 5'-TGAAGGGGTCATTGATGGCAACA-3' \\
U6 & 5'-CTCGCTTCGGCAGCACATA-3 & 5'-CGAATTTGCGTGTCATCCT-3' \\
\hline
\end{tabular}


may suppress cell invasion and migration of malignant melanoma cells.

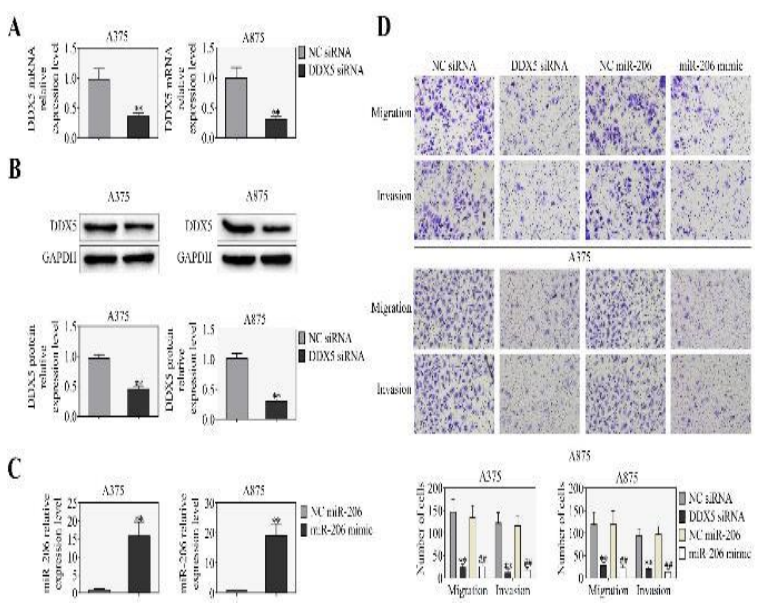

Figure 2: Inhibition of DDX5 or overexpression of miR-206 repressed invasion and migration of A375 and A875 cells. A, Relative mRNA expression of DDX5 was quantified by qPCR after knockdown of DDX5 in A375 and A875 cells. B, DDX5 protein expression was quantified by western blot after knockdown of DDX5 in A375 and A875 cells. C, Relative mRNA expression miR-206 was quantified by qPCR after overexpression of miR-206 in A375 and A875 cells. D, Cell invasion and migration were measured by transwell assay after knockdown of DDX5 or overexpression of miR-206 in A375 and A875 cells. ${ }^{* *} p<0.01$, compared to NC siRNA. ${ }^{\# \#} p<$ 0.01 , compared to NC miR-206.

\section{MiR-206 repressed expression of cytoskeletal proteins}

To investigate the effects of miR-206 and DDX5 on cytoskeleton remodeling, protein and mRNA levels of the cytoskeletal proteins vinculin, coronin3, and ezrin were determined after knockdown of DDX5 or overexpression of miR206. Vinculin, coronin3, and ezrin levels were lower after knockdown of DDX5 in A375 and A875 cells $(p<0.01$; Figure 3A, B). Vinculin, coronin3, and ezrin levels were also lower following overexpression of miR-206 in A375 and A875 cells $(p<0.01$; Figure 4A, B). These findings suggest that inhibition of DDX5 or overexpression of miR-206 inhibits expression of cytoskeletal proteins.

\section{Overexpression of DDX5 reverses the effect of miR-206 on cytoskeletal proteins}

Relative expression levels of vinculin, coronin3 and, ezrin were downregulated upon overexpression of miR-206, but upregulated upon overexpression of DDX5 and miR-206 in A375 and A875 cells when compared the to control $(p<0.01$; Figure 5A). Western blot analysis also showed that protein expression of vinculin, coronin3, and ezrin decreased upon overexpression of miR-206, but increased upon overexpression of DDX5 and miR-206 in A375 and A875 cells when compared to the control ( $p$ $<0.01$; Figure 5B). Thus, overexpression of DDX5 reversed the effect of miR-206 overexpression on cytoskeletal protein levels.

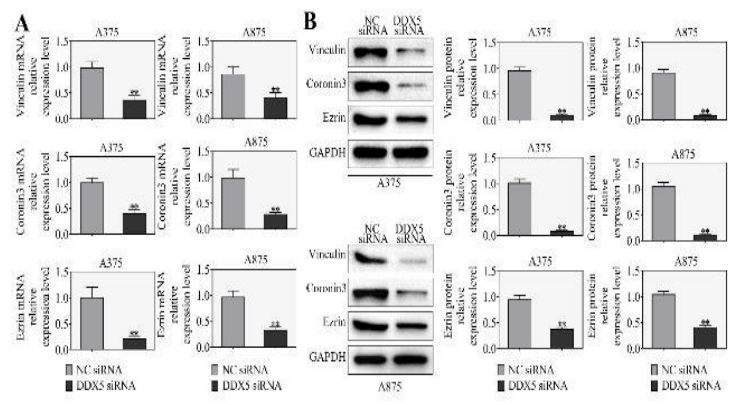

Figure 3: Knockdown of DDX5 MiR-206 repressed expression of cytoskeletal proteins in A375 and A875 cells. A, Relative expression levels of vinculin, coronin3 and ezrin were quantified after knockdown of DDX5 in A375 and A875 cells. B, Protein expression of vinculin, coronin3, and ezrin was quantified after knockdown of DDX5 in A375 and A875 cells. ${ }^{\text {** }} p<$ 0.01 , compared to NC siRNA

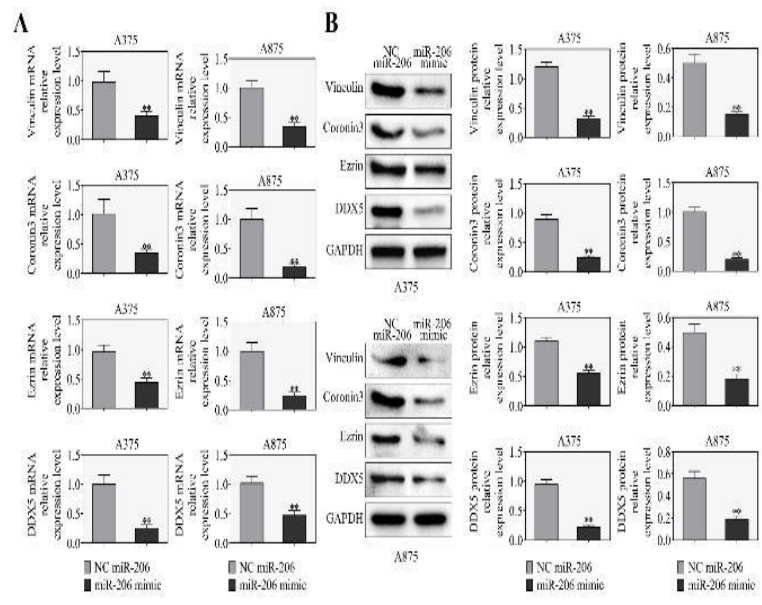

Figure 4: MiR-206 repressed expression of cytoskeletal proteins in A375 and A875 cells. A, Relative expression levels of vinculin, coronin3, and ezrin were quantified following overexpression of miR206 in A375 and A875 cells. B, Protein expression of vinculin, coronin3, and ezrin was quantified following overexpression of miR-206 in A375 and A875 cells. ${ }^{* *} p$ $<0.01$, compared to NC miR-206

\section{MiR-206 directly targets DDX5}

Pairing between miR-206 and DDX5 sequences was predicted to occur between the DDX5 WT 3'UTR sequence ACAUUCC and the miR-206 sequence UGUAAGG (Figure 6A). The luciferase reporter assay confirmed that overexpression of 
miR-206 inhibited expression of the $3^{\prime}$-UTR of DDX5-WT, but not of the 3'-UTR of DDX5-MUT, as observed by luciferase activity $(p<0.01$; Figure $6 \mathrm{~B})$, indicating that miR-206 directly targets DDX5.

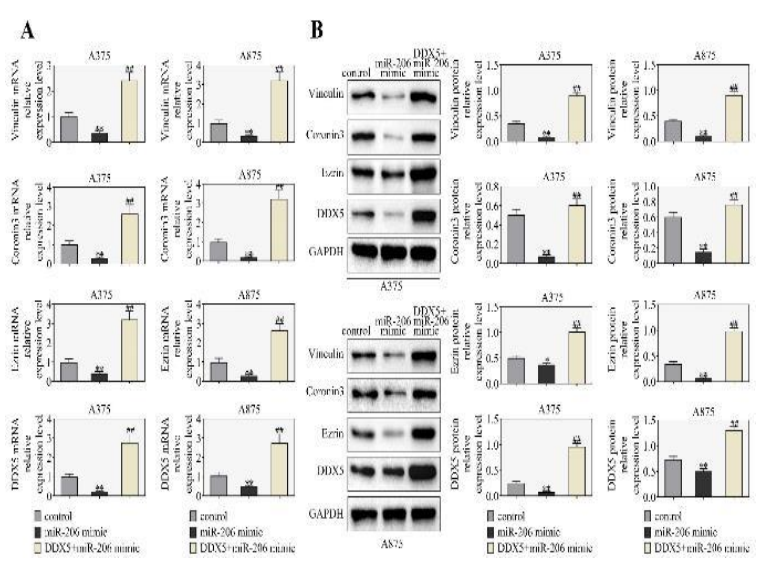

Figure 5: Overexpression of DDX5 increased cytoskeletal protein levels in A375 and A875 cell lines. A, Relative expression levels of vinculin, coronin3, and ezrin were quantified upon overexpression of miR-206 and DDX5 in A375 and A875 cells. B, Protein expression of vinculin, coronin3, and ezrin was quantified upon overexpression of miR-206 and DDX5. ${ }^{* *} p<0.01$, compared to the control. ${ }^{\# \#} p<0.01$, compared to the miR-206 mimic

A

$\begin{array}{ll}\text { DDX5-WT } & 5 \text {,...GUUGGAUAUUUCUCUACAUUCCU...3', } \\ \text { hsa-miR-206 } & \text { 3, GGUGUGUG } \triangle \wedge \text { GG } \wedge \wedge \text { UGUAAGGU } \\ \text { DDX5-MUT } & 5,\end{array}$

B

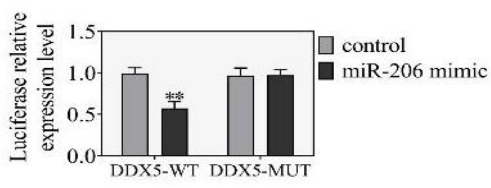

Figure 6: DDX5 is a direct target of miR-206. A, Pairing between miR-206 and 3'-UTR DDX5-WT sequences. B, The miR-206 mimic inhibited expression of the 3'-UTR of DDX5-WT as observed by luciferase activity. ${ }^{* *} p<0.01$, compared to the control

\section{DISCUSSION}

Cell migration plays a key role in tumor invasion and metastasis, and the control of cell migration may provide a promising strategy for the eradication of cancer [12]. Increasing evidence suggests that actin reorganization is a major factor in cell motility and is critical for most types of cell migration [12]. Thus, targeting cytoskeleton reorganization may be an antimetastatic remedy. In this study, the role and mechanism of miR-206 on cytoskeleton remodeling in melanoma cells were investigated. The results indicate that miR-206 represses cytoskeleton reorganization via inhibition of DDX5 expression in melanoma cells.

It was reported that repression of miR-206 leads to abnormal cell proliferation and migration, which contributes to the development of nonsmall cell lung cancer [13]. In addition, evidence showed that reduced serum miR-206 levels associate with poor prognosis in patients with melanoma [14]. Recently, transfection of breast cancer cells with miR-206 mimics inhibited cell invasion and migration through regulation of actin cytoskeleton remodeling [6]. This study revealed that reduced miR-206 levels significantly suppressed cell invasion and migration of A375 and A875 cells. miR-206 also repressed expression of the cytoskeletal proteins, vinculin, coronin3, and ezrin.

A previous study demonstrated that DDX5 promotes cell migration and metastasis [15]. It was also shown that DDX5 may function as a target for suppression of cell migration and invasion of basal cell carcinoma via inhibition of the JAK2/STAT3 pathway [16]. More importantly, DDX5 was shown to be involved in actin cytoskeleton reorganization in breast cancer cells [17]. These findings indicated that DDX5 contributes to the conformational changes in migrating cells [18]. In this study, elevated DDX5 levels were observed in A375 and A875 cells. Overexpression of DDX5 significantly increased vinculin, coronin3, and ezrin expression, and knockdown of DDX5 reduced expression of these cytoskeletal proteins and inhibited cell invasion and migration. These results revealed that DDX5 may promote cytoskeletal reorganization in A375 and A875 cells and that inhibition of DDX5 may repress reorganization of the cytoskeleton and cell invasion and migration of melanoma cells.

To investigate the relationship between miR-206 and DDX5 in cytoskeleton reorganization, target genes of miR-206 were predicted and analyzed by luciferase reporter assay. A sequence complementary to a sequence in miR-206 was found in the 3'-UTR of WT DDX5, indicating that DDX5 is a target gene of miR-206, and this was confirmed by luciferase assay. This study is the first to investigate the effect of miR-206 on cytoskeleton remodeling in melanoma cells in vitro. The results of this study need to be confirmed in vivo.

\section{CONCLUSION}

MiR-206 suppresses reorganization of the cytoskeleton in melanoma cells. Therefore, it is 
potential target for an anticancer therapy for melanoma.

\section{DECLARATIONS}

\section{Acknowledgement}

This study was supported by the Foundation of Research on Technology and Quality Standards of Mixture Formula for Regulating Stomach and Releasing Excessive Turbid (no. ZRQN1509, 030029031 to Wei Jin).

\section{Conflict of Interest}

There are no conflicts of interest to disclose.

\section{Availability of data and materials}

All data generated or analyzed during this study are included in this published article.

\section{Contribution of authors}

We declare that this work was done by the authors named in this article and all liabilities pertaining to claims relating to the content of this article will be borne by the authors. ShengLin Wu designed the study and supervised the data collection. Shan Nie analyzed and interpreted the data. Jian Wang, Liping Ye, Xiaoen You and Xuefeng Zhu prepared the manuscript for publication and reviewed the draft of the manuscript. All authors have read and approved the manuscript.

\section{Open Access}

This is an Open Access article that uses a funding model which does not charge readers or their institutions for access and distributed under the terms of the Creative Commons Attribution License (http://creativecommons.org/licenses/by/ 4.0) and the Budapest Open Access Initiative (http://www.budapestopenaccessinitiative.org/rea d), which permit unrestricted use, distribution, and reproduction in any medium, provided the original work is properly credited.

\section{REFERENCES}

1. Berwick $M$, Buller $D B$, Cust $A E$, Gallagher RP, Lee TK, Meyskens FL, Pandey S, Thomas NE, Veierod MB, Ward S. Melanoma Epidemiology and Prevention. Cancer Treat Res 2016; 167: 17-49.

2. Melanoma research gathers momentum. Lancet (London, England) 2015; 385(9985): 2323.
3. Liang H, Zhao Y, Pi J, Luo R. MiR-875-5p suppresses cervical cancer cell proliferation and metastasis via negative regulation of EGFR. Trop J Pharm Res 2021; 20(5): 939-946.

4. Tsang FH, Au SL, Wei L, Fan DN, Lee JM, Wong CC, Ng IO, Wong C. MicroRNA-142-3p and microRNA-142-5p are downregulated in hepatocellular carcinoma and exhibit synergistic effects on cell motility. Front Med 2015; 9(3): 331-343.

5. Wang J, Tsouko E, Jonsson P, Bergh J, Hartman J, Aydogdu E, Williams C. miR-206 inhibits cell migration through direct targeting of the actin-binding protein Coronin $1 C$ in triple-negative breast cancer. Mol Oncol 2014; 8(8): 1690-1702.

6. Liu H, Cao Y, Ye W, Sun Y. Effect of microRNA-206 on cytoskeleton remodelling by downregulating Cdc42 in MDA-MB-231 cells. Tumori 2010; 96(5): 751-755.

7. Nyamao RM, Wu J, Yu L, Xiao X, Zhang F. Roles of $D D X 5$ in the tumorigenesis, proliferation, differentiation, metastasis and pathway regulation of human malignancies. Biochim Biophys Acta 2019; 1871(1): 8598.

8. Clark EL, Coulson A, Dalgliesh C, Rajan P, Nicol SM, Fleming $S$, Heer R, Gaughan L, Leung HY, Elliott DJ. The RNA helicase p68 is a novel androgen receptor coactivator involved in splicing and is overexpressed in prostate cancer. Cancer Res 2008; 68(19): 7938-7946.

9. Wortham NC, Ahamed E, Nicol SM, Thomas RS, Periyasamy M, Jiang J, Ochocka AM, Shousha S, Huson L, Bray SE. The DEAD-box protein $p 72$ regulates ERo-/oestrogen-dependent transcription and cell growth, and is associated with improved survival in ERapositive breast cancer. Oncogene 2009; 28(46): 40534064.

10. Wang D, Huang J, Hu Z. RNA Helicase DDX5 Regulates MicroRNA Expression and Contributes to Cytoskeletal Reorganization in Basal Breast Cancer Cells. Mol Cell Proteomics 2012; 11(2).

11. Livak KJ, Schmittgen TD. Analysis of relative gene expression data using real-time quantitative $P C R$ and the 2(-Delta Delta C(T)) Method. Methods 2001; 25(4): 402-408.

12. Yamazaki D, Kurisu S, Takenawa T. Regulation of cancer cell motility through actin reorganization. Cancer Sci 2005; 96(7): 379-386.

13. Sun C, Liu Z, Li S, Yang C, Xue R, Xi Y, Wang L, Wang $S$, He Q, Huang J, et al. Down-regulation of c-Met and Bcl2 by microRNA-206, activates apoptosis, and inhibits tumor cell proliferation, migration and colony formation. Oncotarget 2015; 6(28): 25533-25574.

14. Tian R, Liu T, Qiao L, Gao M, Li J. Decreased serum microRNA-206 level predicts unfavorable prognosis in patients with melanoma. Int J Clin Exp Pathol 2015; 8(3): 3097-3103.

15. Wang $H$, Gao X, Yang J, Liu Z. Interaction between $p 68$ RNA helicase and $\mathrm{Ca}^{2+}$-calmodulin promotes cell migration and metastasis. Nat Commun 2013; 4: 1354. 
16. Quan Z, Zhang B, Yin F, Du J, Zhi Y, Xu J, Song N. DDX5 Silencing Suppresses the Migration of Basal cell Carcinoma Cells by Downregulating JAK2/STAT3 Pathway. Technol Cancer Res Treat 2019; 18: 1533033819892258.

17. Wang $D$, Huang J, Hu Z. RNA helicase DDX5 regulates microRNA expression and contributes to cytoskeletal reorganization in basal breast cancer cells. Mol Cell Proteomics 2012; 11(2): M111.011932.

18. Dai T, Cao L, Yang Z, Li Y, Tan L, Ran X, Shi C. P68 RNA helicase as a molecular target for cancer therapy. J Exp Clin Cancer Res 2014; 33: 64. 